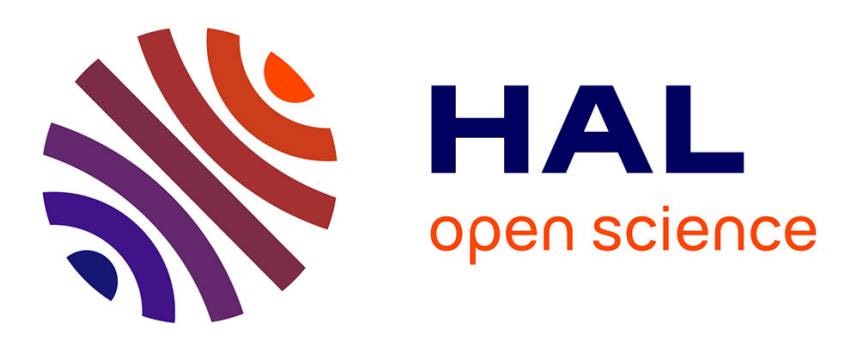

\title{
Les conséquences de la dynamique de la digestion des aliments sur le métabolisme ruminal et les performances animales
}

\author{
Daniel Sauvant, Jaap J. van Milgen
}

\section{- To cite this version:}

Daniel Sauvant, Jaap J. van Milgen. Les conséquences de la dynamique de la digestion des aliments sur le métabolisme ruminal et les performances animales. Productions Animales, 1995, 8 (5), pp.353-367. hal-00896132

\section{HAL Id: hal-00896132 \\ https://hal.science/hal-00896132}

Submitted on 1 Jan 1995

HAL is a multi-disciplinary open access archive for the deposit and dissemination of scientific research documents, whether they are published or not. The documents may come from teaching and research institutions in France or abroad, or from public or private research centers.
L'archive ouverte pluridisciplinaire HAL, est destinée au dépôt et à la diffusion de documents scientifiques de niveau recherche, publiés ou non, émanant des établissements d'enseignement et de recherche français ou étrangers, des laboratoires publics ou privés. 
INRA Prod. Anim., $1995,8(5), 353-367$
D. SAUVANT, J. VAN MILGEN*

INRA-INAPG Laboratoire de Nutrition et Alimentation 16 rue Claude Bernard 75231 Paris Cedex 05

*INRA Station de Recherches Porcines 35590 Saint Gilles

\section{Les conséquences de la dynamique de la digestion des aliments sur le métabolisme ruminal et les performances
animales}

Au cours des dernières années les travaux de recherche ont montré que les différentes fractions des aliments étaient dégradées dans le réticulo-rumen selon des vitesses variant largement d'un aliment, ou d'une fraction, à l'autre. Sur la base de ces résultats, et du fait que l'azote et l'énergie peuvent être facteur limitant de la croissance microbienne dans le réticulo-rumen, il s'est petit à petit dégagé le concept de formulation de régimes ou d'aliments "synchrones". Ce type de formulation a pour but de fournir de l'azote et de l'énergie disponible de manière continue et dans une proportion adaptée aux besoins des microbes de la panse. Différentes études expérimentales ont été mises en place pour valider ce concept, il paraît donc utile de faire le point de ces connaissances récentes pour juger de l'opportunité de la formulation de ces régimes qualifiés de « synchrones".

\section{Résumé}

Les travaux des dernières décennies ont montré que les cinétiques de dégradation ruminale des aliments variaient largement en fonction de leur nature et du constituant considéré. Il est donc envisageable de mettre à profit cette variabilité pour formuler des aliments composés, ou des régimes mixtes, plus ou moins rapidement dégradables ou plus ou moins bien équilibrés entre les flux des glucides et des protéines disponibles par rapport aux besoins des micro-organismes du rumen.

Les résultats expérimentaux sur les effets des variations de ces phénomènes sur la digestion ruminale et sur les performances zootechniques indiquent que ces effets sont beaucoup moins importants que ce qui pouvait être envisagé a priori. Il est de ce fait nécessaire de rechercher des explications sur la capacité du rumen à " amortir " ces variations. Il semble que ce soit l'existence de nombreuses structures et phénomènes de délais qui permettent d'amortir efficacement les variations dynamiques des processus de dégradation entre aliments et constituants. Plusieurs exemples sont évoqués. Pour les constituants azotés, les phénomènes d'amortissement existent en particulier au niveau du stockage transitoire de molécules peptidiques avant captation par les cellules microbiennes et des recyclages d'azote par les protozoaires et le recyclage d'urée d'origine sanguine. Pour les constituants énergétiques, il semble que le stockage transitoire d'énergie le plus important soit le compartiment polysaccharidique des microbes. L'approche dynamique des phénomènes digestifs ruminaux indique en outre que la croissance microbienne est limitée et peu adaptable dans le court terme même si une quantité suffisante de nutriments est disponible. Quelques commentaires sont effectués sur cet aspect.
Les productions des ruminants sont, comme les autres productions animales, confrontées à un ensemble d'objectifs et de contraintes de plus en plus diversifiés. En effet, outre la recherche d'une meilleure productivité, il faut aujourd'hui prendre en compte un ensemble d'autres aspects tels que la qualité des produits commercialisés, le respect de l'environnement, le bien-être de l'animal d'élevage... Cette diversification des préoccupations a logiquement amené l'émergence du concept de « lois de réponse »de l'animal aux variations de son régime alimentaire. Ce concept vient en fait compléter l'approche classique qui consistait à formuler un régime alimentaire dans le but unique de satisfaire les différents besoins nutritifs (énergie, protéines, minéraux...) liés à la réalisation du potentiel de production d'un animal donné.

L'application pratique du concept de loi de réponse nécessite l'identification et l'intégration de tous les processus susceptibles de jouer un rôle déterminant. Cela implique en 
particulier d'intégrer certains mécanismes biologiques sous-jacents et revient à étudier les réponses à l'alimentation des organes qui déterminent le plus les réponses zootechniques évoquées ci-dessus. Chez le ruminant, le réticulo-rumen joue à l'évidence un tel rôle. En effet, il est aisé de montrer que le fonctionnement de cet organe est déterminant vis-à-vis de la digestibilité de la ration, donc de sa valeur énergétique, vis-à-vis des flux de rejets fécaux de matière organique, urinaires d'azote, et gazeux de $\mathrm{CO}_{2}$ et de $\mathrm{CH}_{4}$. Enfin, de nombreuses études ont montré que les variations des conditions digestives ruminales étaient susceptibles de moduler la composition des produits élaborés (carcasses, lait...).

La compréhension et la prévision des lois de réponse nécessitent également de pouvoir raisonner autrement que sur la base temporelle journalière habituelle. Il faut en effet pouvoir intégrer, si nécessaire, d'une part des phénomènes dynamiques de court terme, basés sur des périodes allant de quelques minutes à quelques heures et, d'autre part, des phénomènes de long terme s'étendant sur des périodes allant de plusieurs semaines à quelques mois. A ce propos il est bien connu que les phénomènes digestifs ruminaux sont rythmés par des phénomènes associés à des périodes ou des pas de temps bien inférieurs à 24 heures. Les principales raisons sont d'une part la caractéristique dynamique des repas qui se traduit par des entrées irrégulières de substrats dans le réticulo-rumen et, d'autre part, les variations de la vitesse de dégradation des aliments sous l'action des microbes. L'objet de cet article est d'envisager les principales conséquences nutritionnelles et pratiques de ces aspects dynamiques.

\section{1 / Aspects dynamiques de la digestion des aliments}

\section{1 / Méthodes d'étude}

Au cours des dernières décennies, le développement de la méthode in situ, ou in sacco, qui consiste à faire incuber dans le rumen des aliments emprisonnés dans un sachet de

Tableau 1. Equations d'ajustement des cinétiques de dégradation des constituants alimentaires dans le réticulo-rumen.
Dégradation sans temps de latence:
Dégradation avec temps de latence $L$ :
Dégradation + transit, sans temps de latence:
Dégradation + transit, avec temps de latence:
$D t$ : dégradation à l'instant $t$
DT : dégradation théorique ( effective degradability ")
fs : fraction soluble
fd : fraction insoluble dégradable
$\mathrm{kd}$ : vitesse relative de dégradation de la fraction $\mathrm{fd}(/ \mathrm{h})$
$\mathrm{kp}$ : vitesse relative de transit $(/ \mathrm{h})$
$\mathrm{L}$ : temps de latence discret avant digestion $(\mathrm{h})$
$\mathrm{t}$ : temps d'incubation $(\mathrm{h})$

$D t=f s+f d\left(1-e^{-k d t}\right)$

$D t=f s+f d\left(1-e^{-k d(t-L)}\right)$

$\mathrm{DT}=\mathrm{fs}+\mathrm{fd}[\mathrm{kd} /(\mathrm{kd}+\mathrm{kp})]$

$D T=f s+f d[k d /(k d+k p)] e^{-k p L}$ nylon, a permis d'améliorer sensiblement les connaissances sur les cinétiques de dégradation des constituants alimentaires. La façon la plus simple d'interpréter et de modéliser, par ajustement statistique, les cinétiques de dégradation consiste à supposer que l'aliment, ou bien un de ses constituants, se compose de 3 fractions majeures: une soluble (fs), une potentiellement digestible (fd), et une indigestible $(\mathbf{f i}=1-\mathrm{fs}-\mathrm{fd})$. La fraction $\mathbf{f s}$ est supposée être mise directement à disposition et utilisée par les micro-organismes, tandis que la fraction fd est progressivement dégradable suivant une vitesse relative de dégradation supposée constante $(k d)$. Le phénomène réel est en fait bien plus compliqué. En particulier, il existe souvent une phase de latence (tableau 1) avant la mise en place du processus de dégradation de fd et, d'autre part, le taux de dégradation kd n'est pas toujours constant pour un même constituant. L'avantage de l'ajustement des cinétiques de dégradation par des modèles simples est notamment lié au fait qu'il est ainsi possible d'intégrer assez simplement (tableau 1) l'influence du transit des particules pour calculer la "dégradabilité théorique " ( effective degradability » des anglo-saxons).

\section{2 / Les protéines}

Les méthodes in situ ont été principalement appliquées à la fraction azotée des aliments. Les résultats ont été largement utilisés lors de la mise au point des systèmes modernes d'unités d'alimentation protéique des ruminants (Madsen 1985, Vérité et al 1987). Dans ces approches les 3 fractions évoquées ci-dessus ont été distinguées alors que 5 fractions ont été proposées dans les systèmes de Cornell (Sniffen et al 1992). Il reste cependant à démontrer l'intérêt de la distinction de 5 fractions au lieu de 3 . Les approches in situ ont permis de hiérarchiser les matières premières, donc de leur attribuer des valeurs alimentaires individualisées. Cela a par exemple permis de prendre en compte l'influence du niveau de fertilisation d'un fourrage, de l'état de conservation de l'ensilage et de différents traitements technologiques. Il y a en général une assez bonne cohérence entre les flux des protéines alimentaires non dégradées passant au duodénum (PIA) et ceux qui sont prédits à partir des cinétiques de dégradation in sacco et des estimations du transit des particules (tableau 1). C'est sur cette cohérence qu'ont été établies les équations de base d'un système tel que le système PDI.

\section{3 / Les glucides}

La dégradation des fractions glucidiques des aliments a été moins étudiée que celle des protéines. On considère que les glucides solubles sont immédiatement disponibles pour les micro-organismes. Cette hypothèse est assez cohérente avec l'estimation d'un taux de dégradation de $2,5 \%$ par minute faite dans le système de Cornell. Cependant, 
il apparaît fréquemment que la teneur en glucides solubles du jus de rumen n'est pas négligeable aussitôt après un repas. Au cours des dernières années, différentes publications ont permis d'améliorer les connaissances sur les cinétiques de dégradation de l'amidon. Sur cet aspect nous renvoyons les lecteurs à un article publié dans cette même revue (Sauvant et al 1994). Rappelons que ces cinétiques de dégradation peuvent être très variables suivant l'aliment ou le type d'amidon considéré. Ceci permet notamment de faire une typologie des aliments et de distinguer les ingrédients riches en amidon facilement soluble et dégradable (orge, avoine, ...) des ingrédients à amidon peu soluble et lentement dégradable (maïs, sorgho...). D'autre part il est connu que des traitements technologiques hydrothermiques peuvent accroître la dégradabilité de l'amidon d'un ingrédient à " amidon lent». Enfin des études récentes ont montré que l'amidon de blé, qui est du type rapide, peut être "protégé " par un traitement au formaldéhyde ( $\mathrm{Mc}$ Allister et al 1989, Chapoutot et al non publié). Il est possible d'estimer, à l'aide d'une équation de régression, les quantités d'amidon qui sont respectivement dégradable et non dégradable dans le réticulo-rumen. Il est également possible d'aboutir à une estimation de la quantité d'amidon protégé qui est digéré dans l'intestin. Les éléments de cette démarche ont été décrits par Sauvant et al (1994).

Figure 2. Teneur et vitesse de dégradation de la paroi végétale des fourrages (Sauvant et al 1994).

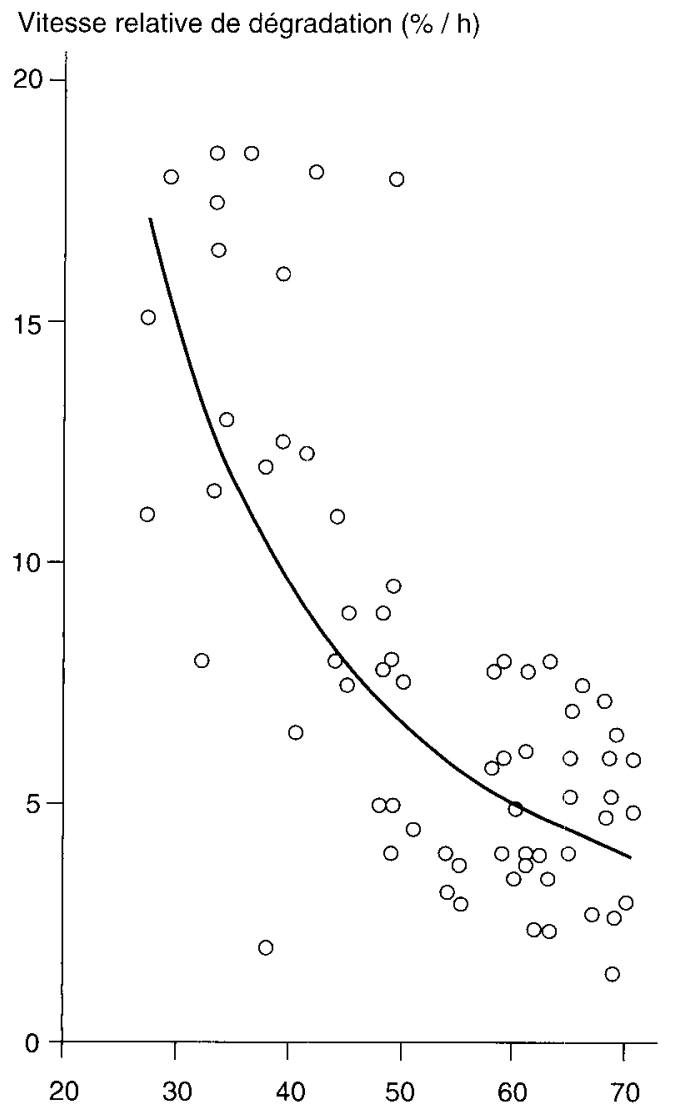

Teneur en parois végétales (NDF, en \% MS)
Figure 1. Cinétique de dégradation in situ des glucides structuraux ( $P$. Chapoutot et al, non publié).

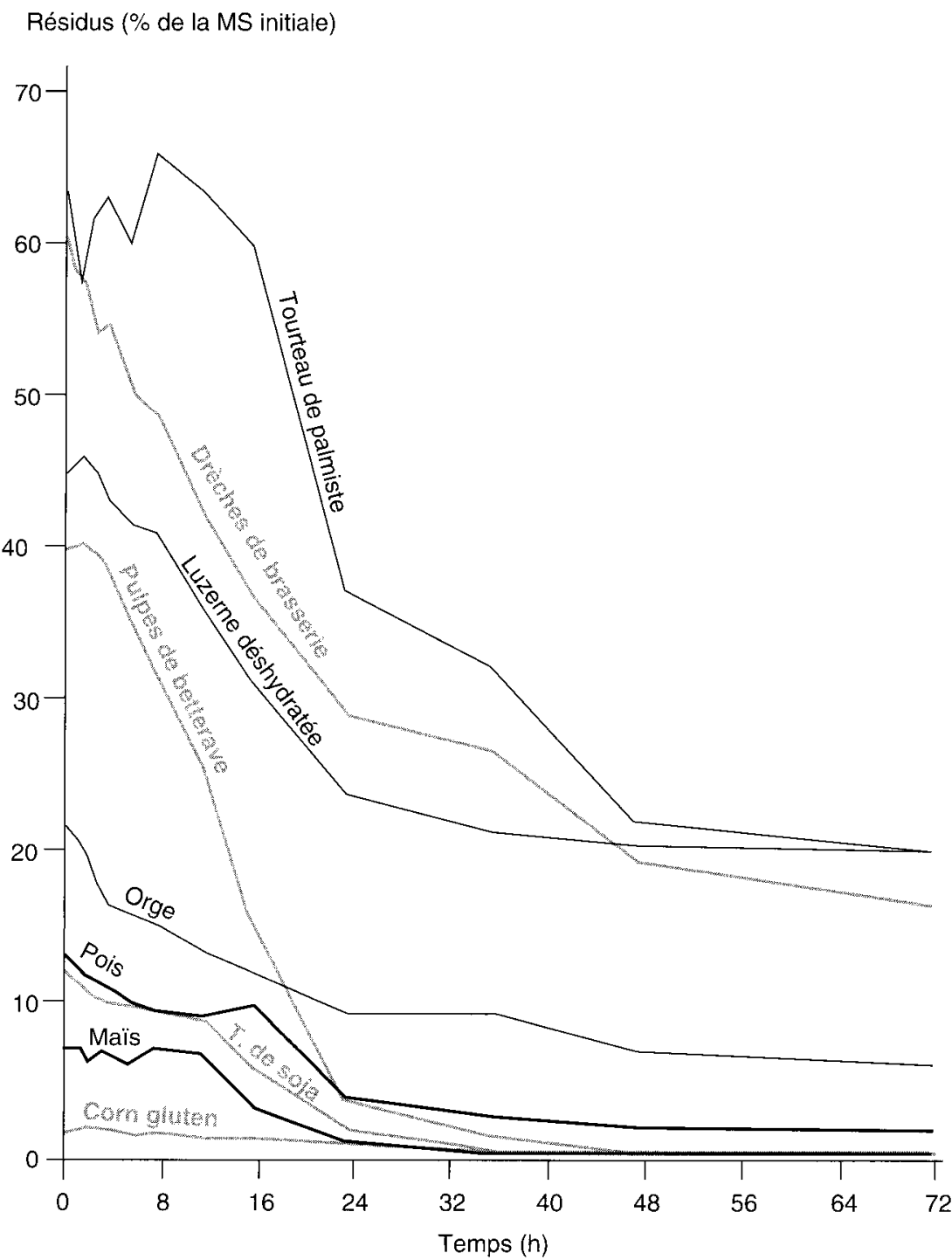

Les cinétiques de dégradation des glucides structuraux (cellulose et hémicellulose) diffèrent de celles des glucides non structuraux et sont plus variables (figure 1). Ces cinétiques se caractérisent notamment par :

- une fraction indigestible fi importante, en particulier pour les fourrages et certains sous-produits. Cette fraction fi est principalement liée à la lignification de la paroi (Jarrige 1981, Sauvant et al 1989).

- un temps de latence qui peut atteindre in situ une dizaine d'heures avant que le processus de dégradation ne se mette en route. Ce phénomène peut altérer fortement la digestibilité ruminale des parois comme indique l'équation (4) du tableau 1.

- une forte irrégularité de la vitesse de dégradation (Chapoutot et al 1993).

La vitesse moyenne de dégradation des glucides pariétaux est, pour les fourrages, négativement liée à la teneur en paroi (figure 2). Ceci montre que la quantité de paroi dégradée des fourrages par unité de temps tend à être constante lorsqu'elle est exprimée par $\mathrm{kg}$ de MS ou de MO du fourrage. Par contre il 
n'y a pas de relation au sein des aliments concentrés et sous-produits entre ces 2 paramètres. Cette absence de relation s'explique en particulier par la grande diversité d'organes et d'espèces végétales utilisés comme concentrés et sous-produits. Il convient par ailleurs de rappeler que le processus de dégradation des glucides structuraux est particulièrement sensible aux conditions écologiques ruminales, en particulier au $\mathrm{pH}$. Cette sensibilité constitue la principale origine des phénomènes d'interactions digestives négatives (Michalet-Doreau et al 1993). Les études de validation de la dégradabilité in situ à l'aide des digestibilités duodénales sont rares. Cependant les quelques résultats récents disponibles (Archimède et al 1995, Martin 1994) indiquent que la dégradation in situ des constituants pariétaux serait bien moins efficace qu'in vivo. Ceci pourrait révéler de fortes interactions digestives dans les sachets de nylon.

\section{2 / Implications nutritionnelles et pratiques des cinétiques de dégradation}

\section{1 / Le concept d'harmonisation de dégradation des glucides et protéines}

Les résultats accumulés sur les cinétiques de dégradation in situ des glucides et protéines ont permis de démontrer l'existence d'une irrégularité de la disponibilité dans le temps des nutriments glucidiques et azotés pour les microbes. En outre, l'équilibre entre les nutriments azotés et glucidiques disponibles peut également largement varier selon l'instant considéré. Un schéma du type de celui de la figure 3 traduit ce principe et a abouti à la recommandation de chercher à combiner les aliments de manière à pouvoir fournir à tout instant le "juste équilibre " entre les nutriments glucidiques et azotés disponibles pour les microbes du réticulorumen. Il convient de souligner que, par rapport à la réalité, la figure 3 est assez naïve dans la mesure où elle discrimine trop les différents pics, en particulier le flux de glucides disponibles issus des constituants pariétaux peut être considéré comme quasiment continu d'après les simulations que nous avons pu faire à partir d'un modèle mécaniste du rumen adapté pour les événements de court terme (figure 4 ; Sauvant et al, non publié).

Sur la base de ce principe il a été possible d'imaginer des méthodes de calculs de régimes alimentaires " harmonisés » ou " synchronisés " (Sauvant et Giger 1987). Ultérieurement, différentes propositions de formulations de régimes sur la base de ce principe ont été faites, en particulier aux Etats-Unis (Nocek et al 1990). Il est également possible de calculer un index de synchronicité intégré pour évaluer dans quelle mesure une ration s'éloigne ou pas du juste équilibre pour les microbes, qui serait de l'ordre de $25 \mathrm{~g} \mathrm{~N}$ disponible/kg de matière organique disponible, ou $32 \mathrm{~g} \mathrm{~N}$ disponible/kg de glucides disponibles (Sinclair et al 1993).

Figure 3. Aspects théoriques de la dynamique de la disponibilité des glucides et de l'azote dans le rumen.

Les glucides et l'azote ne sont pas disponibles en même temps dans le rumen. On peut donc imaginer de combiner les aliments pour synchroniser leur fourniture aux microbes $d u$ rumen.
Flux dégradé dans le rumen

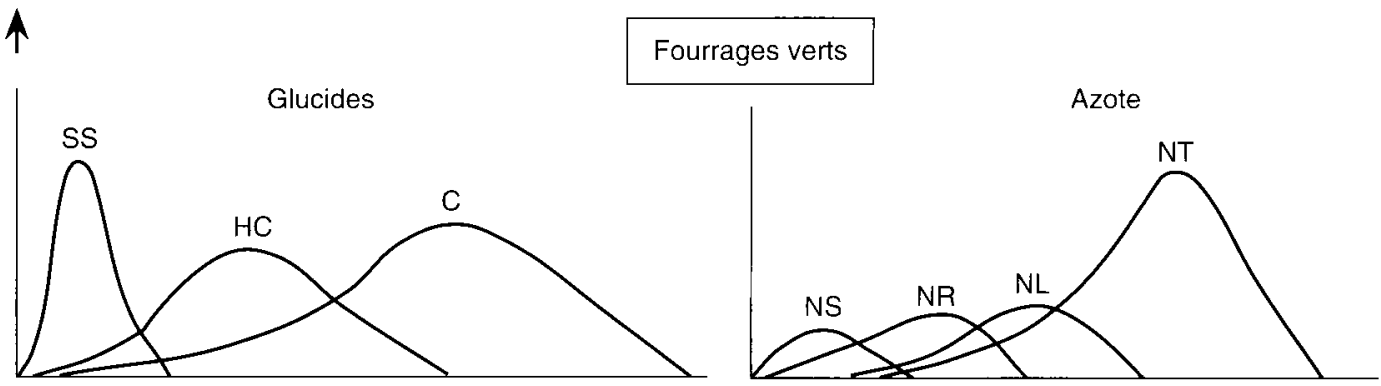

Fourrages ensilés

Glucides

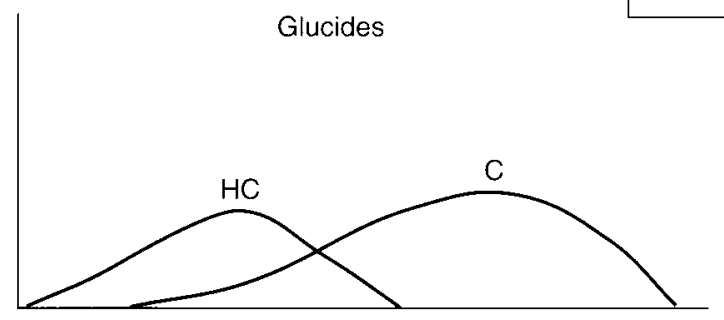

SS : sucres solubles

$\mathrm{HC}$ : hémicelluloses

C : celluloses
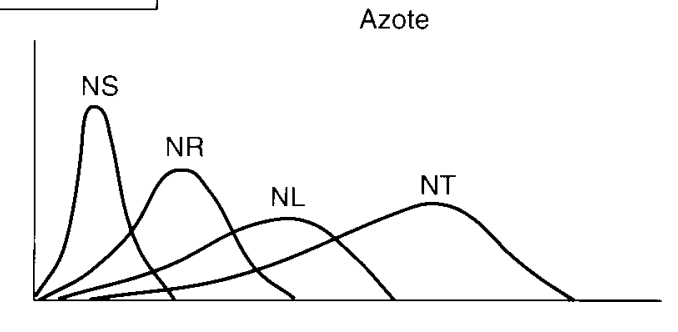

NS : azote soluble

NR : azote rapidement dégradable

NL : azote lentement dégradable

NT : azote très lentement dégradable 
Figure 4. Simulation des flux de carbone associés à une ration riche en aliments concentrés (régime avec $50 \%$ de foin de luzerne $+30 \%$ d'orge $+20 \%$ de tourteau de soja ; $D$. Sauvant, non publié).

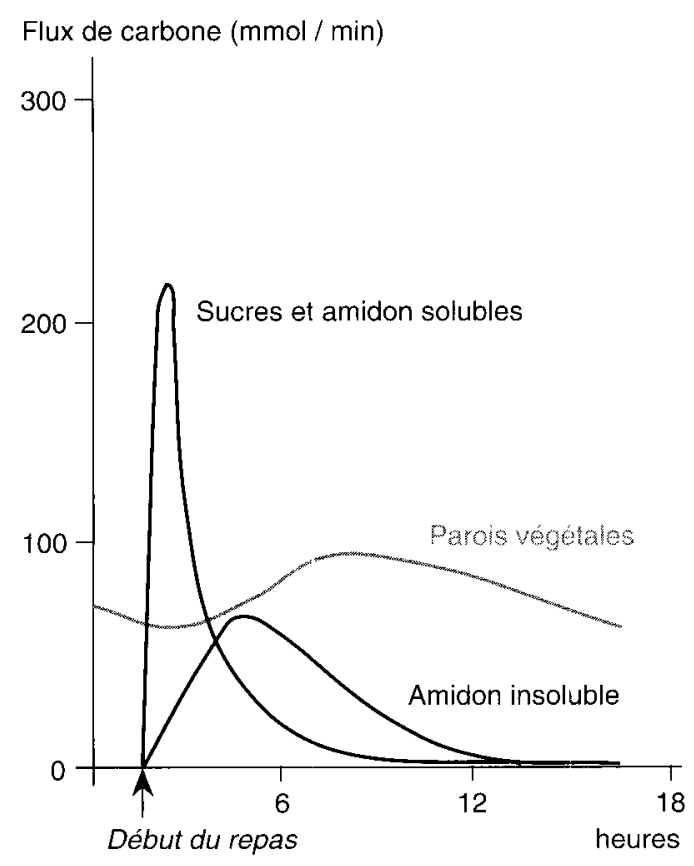

\section{2 / Conséquences sur la digestion ruminale}

\section{a / Dégradation des substrats}

L'opportunité de synchroniser les dégradations des glucides et de l'azote des aliments a été étudiée au laboratoire (Chapoutot et al 1993) in situ, in vitro (Giger-Reverdin et al 1993) sur des mélanges binaires associants factoriellement des sources azotées rapidement (lupin) ou lentement dégradées (glutenmeal) et des sources glucidiques rapidement (orge) ou lentement (maïs) dégradées. L'association de ces ingrédients 2 à 2 a montré que la dégradation des mélanges in situ et in vitro était plus importante que ce que l'on pouvait prévoir à partir des ingrédients simples. Un tel résultat traduit vraisemblablement l'amélioration de l'activité microbienne lorsque des éléments glucidiques et azotés sont simultanément disponibles.

\section{b / Ammoniogénèse}

De nombreuses études ont cherché à quantifier l'influence des composantes dynamiques de la digestion des glucides et de l'azote sur la production d'ammoniac $\left(\mathrm{NH}_{3}\right)$ dans la panse. Le tableau 2 résume les résultats publiés, sachant que, dans tous ces cas, seul l'amidon a été considéré comme constituant glucidique dont la vitesse de dégradation variait. Il apparaît que, pour un niveau donné d'apport alimentaire d'azote, un accroissement de sa vitesse de dégradation augmente significativement la teneur en $\mathrm{NH}_{3}$ du jus de rumen tandis qu'un accroissement de la vitesse de dégradation des glucides présente, pour un même apport azoté, l'effet inverse (tableau 2). Lorsque l'on considère des régimes associant des formes synchrones de glucide et d'azote, il apparaît que la combinaison rapide-rapide libère plus de $\mathrm{NH}_{3}$ que la combinaison lente-lente. Un tel phénomène a également été observé en RUSITEC (GigerReverdin et al, non publié) ; il montre que les microbes présentent une capacité limitée de croissance même si l'azote et les glucides sont disponibles dans les bonnes proportions. Cet aspect important sera discuté ultérieurement. Pour les régimes asynchrones la combinaison "glucide lent-azote rapide " induit une production de $\mathrm{NH}_{3}$ bien plus importante qu'une combinaison " glucide rapide-azote lent " (tableau 2). Ceci veut dire qu'une distinction doit être faite au sein des différents types d'asynchronicité, la combinaison "glucide rapide-azote lent " semble être plus favorable vis-à-vis des microbes et pour l'épargne azotée de l'animal. Il est possible d'extraire de ces données une série de 8 résultats dans lesquels la teneur en $\mathrm{NH}_{3}$ ruminal a été mesurée au sein de dispositifs associant selon un plan factoriel $2 \times 2$ glucide et azote plus ou moins rapidement dégradable. L'interprétation de ces résultats confirme les effets évoqués ci-dessus, elle indique en outre qu'aucune interaction ne peut être détectée entre ces 2 facteurs dont les effets s'additionnent.

\section{c / Profil des acides gras volatils (AGV)}

Une base de 21 résultats publiés sur l'influence de la vitesse de dégradation de l'amidon sur le profil des AGV ont été rassemblés (références fournies à la demande). L'interprétation de ces données laisse apparaître

Tableau 2. Influence d'une combinaison factorielle des vitesses de dégradation des glucides (CHO) et de l'azote $(N)$ sur les paramètres de l'utilisation de l'azote. $n$ : nombre de références (citées dans le texte).

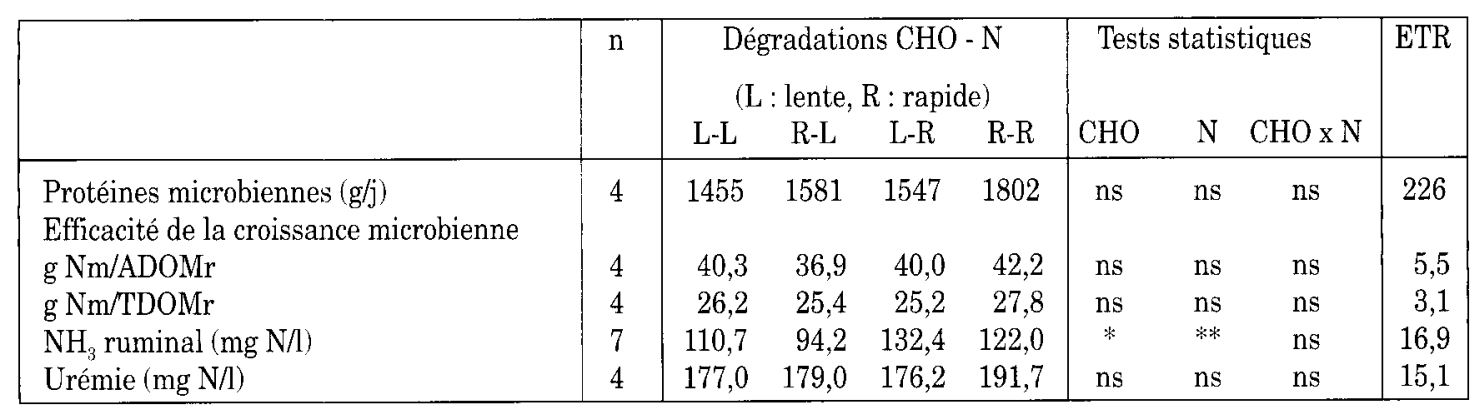

\section{Les régimes synchrones « rapides " conduisent à une concentration ruminale d'NH plus élevée qu'un régime fournissant des glucides rapidement et de l'azote lentement dégradables.}


Figure 5. Influence de la vitesse de dégradation de l'amidon sur la proportion molaire de propionate dans le rumen (Sauvant 1994).

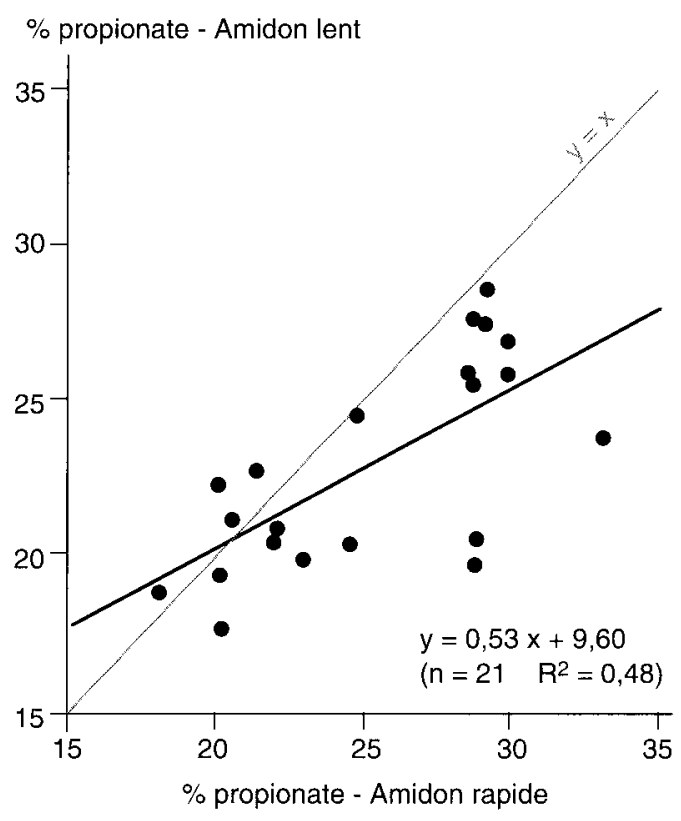

une différence significative Lent-Rapide (L-R) sur les proportions molaires d'acétate $(+1,5 \%)$, de propionate $(-2,3 \%)$ et de butyrate $(+0,6 \%)$. Les figures 5 et 6 indiquent en outre que cette différence dépend de "l'état fermentaire " ruminal. Lorsque cet état fermentaire est normal (proportion de propionate comprise autour de 20 à $25 \%$, figure 5 ; ou rapport acétate/propionate compris entre

Figure 6. Influence de la vitesse de dégradation de l'amidon sur le rapport acétate/propionate dans le rumen.

fonctionnement $d u$ rumen est normal.
3,5 et 4, figure 6) il n'y a pas d'influence de la vitesse de digestion des glucides amylacés. Par contre l'influence devient plus nette lorsque les fermentations ruminales présentent un caractère propionique marqué. Des éléments d'explications de ces résultats seront évoqués dans la partie 3 de ce texte.

\section{d / Protéosynthèse microbienne}

Dans la bibliographie il a été possible d'extraire 16 résultats (références à la demande) montrant qu'une source glucidique lentement dégradable (exemple : maiis grain) réduit de $10,3 \pm 13,2 \%$ le flux d'azote microbien $(\mathrm{Nm})$ au duodénum en comparaison avec une source glucidique rapidement dégradable (exemple : orge). Pour 4 essais combinant factoriellement les taux de dégradation des glucides et de l'azote, cette décroissance est plus importante $(-13,3 \pm 7,2 \%$ vs $-4,5 \pm 12,3 \%)$ quand une source d'azote rapidement dégradable est utilisée. Ces résultats suggèrent qu'une certaine synchronicité entre les vitesses de dégradation serait favorable à la croissance microbienne. Pour 15 résultats, la synthèse microbienne a pu être exprimée par $\mathrm{kg}$ de $\mathrm{MO}$ digérée dans l'ensemble du tube digestif (MODTD). L'emploi d'amidon rapidement dégradable permet d'obtenir une amélioration d'environ $9,5 \mathrm{~g} \mathrm{Nm} / \mathrm{kg}$ MODTD par rapport à de l'amidon rapide (figure 7). Ceci correspond à environ $32 \mathrm{~g}$ PDIM/UF ingérée, ce qui n'est pas négligeable puisque cela représente de l'ordre de $25 \%$ de la valeur PDI d'une ration moyenne pour ruminant laitier. Ces résultats soulignent au passage l'intérêt de chercher à prédire, avec le maximum de précision, la quantité de matière organique fermentée dans le réticulo-rumen (Tamminga et al 1994). Par contre, lorsque la synthèse microbienne a pu être exprimée par kg de matière organique

Figure 7. Influence de la vitesse de dégradation de l'amidon sur la protéosynthèse ruminale rapportée à la matière organique digérée dans l'ensemble du tube digestif ( $g \mathrm{Nm} / \mathrm{kg}$ MOD TD).

Protéosynthèse avec amidon lent

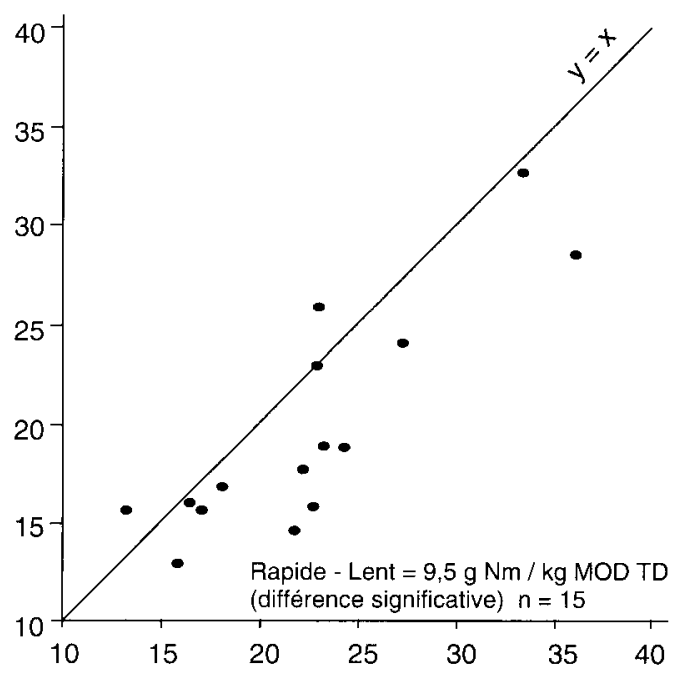

Protéosynthèse avec amidon rapide 
digérée dans le rumen (apparent ou réel) il n'y a pas d'influence de la vitesse de dégradation des glucides et de la source azotée associée (figure 8). Pour 4 de ces 7 publications il est possible de faire une interprétation selon un plan factoriel 2 × 2 (Mc Carthy et al 1989, Herrera-Saldana et Huber 1989, Kung et al 1992, Aldrich et al 1993). L'analyse statistique de ces résultats confirme l'absence d'influence de la vitesse de dégradation de l'amidon et de l'azote ainsi que d'interaction entre ces facteurs sur l'efficacité de la croissance microbienne.

\section{3 / Impact zootechnique}

Au cours des dernières années différents essais ont été conduits pour explorer l'intérêt zootechnique éventuel, en particulier pour des vaches laitières, de formuler des rations ou des concentrés présentant différentes vitesses de dégradation ou/et synchronisant, ou non, les processus de dégradation des glucides et de l'azote. Ainsi la figure 9 montre que le remplacement d'une source d'amidon rapidement dégradable par une lente améliore significativement de $0,79 \pm 1,45 \mathrm{~kg} / \mathrm{j}$ en moyenne la production de lait brut $(\mathrm{n}=18)$. Cependant cette différence L-R disparaît lorsque la production lait est ajustée par le niveau de matière sèche ingérée pris comme une covariable. En effet les animaux recevant des rations riches en amidon rapide ingèrent une moindre quantité de matière sèche. Cette différence pourrait résulter d'une rétroaction homéostasique négative sur l'ingestion plus marquée avec des aliments ou des rations qui

Figure 8. Influence de la vitesse de dégradation de l'amidon sur l'efficacité de la croissance microbienne Noigt et al 1977, Herrera et al 1989, McCarthy et al 1989, Kung et al 1992, Widyobroto 1992, Aldrich et al 1993, Archimède et al 1995).

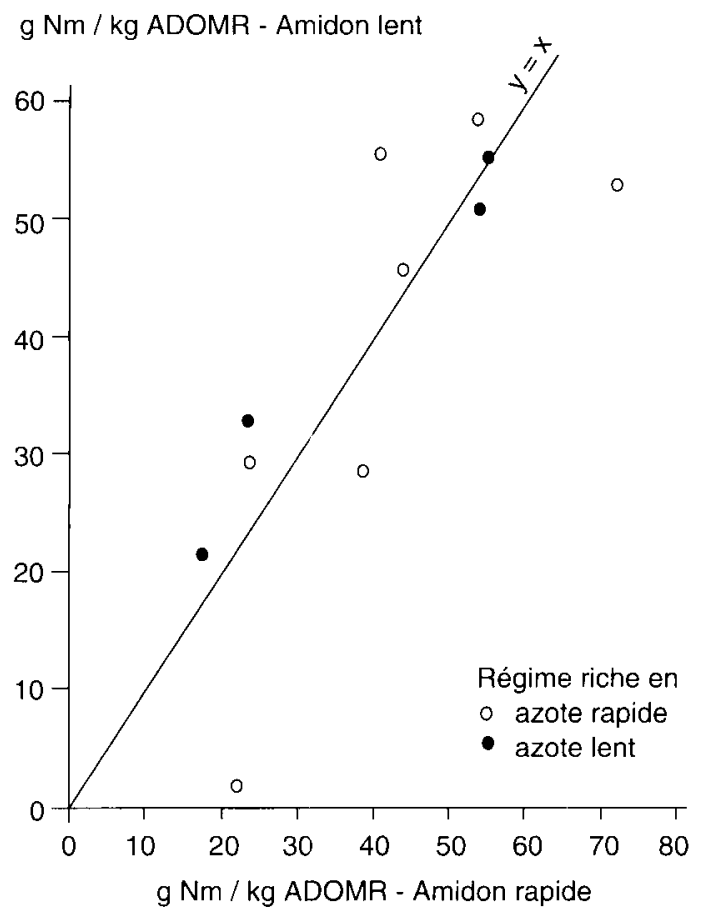

Figure 9. Influence de la vitesse de digestion de l'amidon sur la production laitière de la vache (Sauvant 1994).

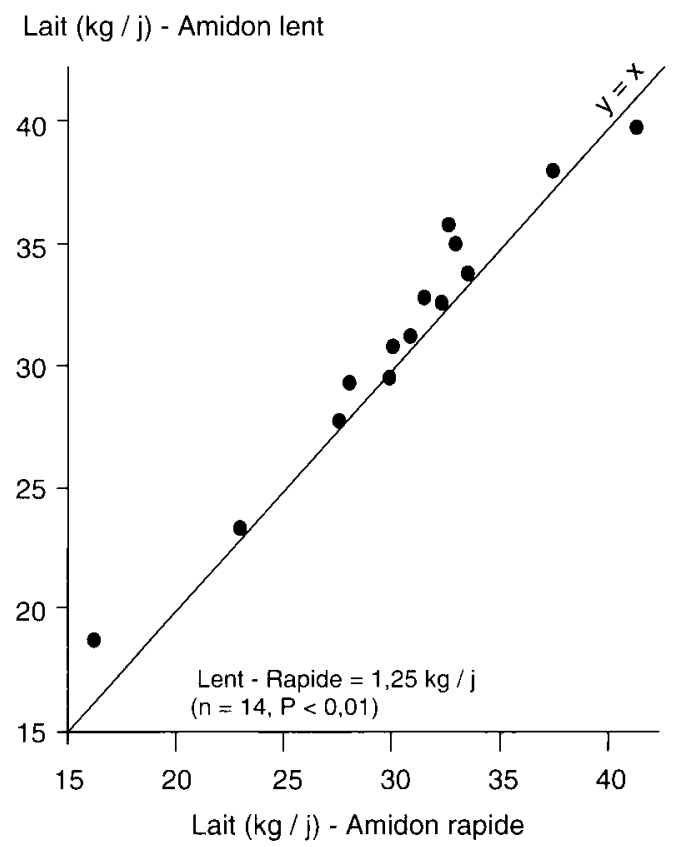

induisent des fermentations ruminales, donc des flux d'AGV absorbés, plus importants. Dans les même essais, la vitesse de dégradation de l'amidon n'a pas eu d'influence sur le taux protéique $\left(\mathrm{TP}_{\mathrm{L}-\mathrm{K}}=-0,31 \pm 0,78 \mathrm{~g} / \mathrm{kg}\right.$, $\mathrm{n}=18)$ et sur le taux butyreux du lait $\left(\mathrm{TB}_{\mathrm{L}-\mathrm{R}}=\right.$ $+1,07 \pm 3,2 \mathrm{~g} / \mathrm{kg}, \mathrm{n}=19$ ). Cependant, comme le montre la figure 10 , la régression du TB présente un coefficient de régression significativement inférieur à 1 . Ceci indique que lorsqu'un régime induit, par sa richesse en énergie, une faible teneur en matières grasses du lait, la vitesse de dégradation de

Figure 10. Influence de la vitesse de digestion de l'amidon sur le taux butyreux du lait de vache (Sauvant 1994).

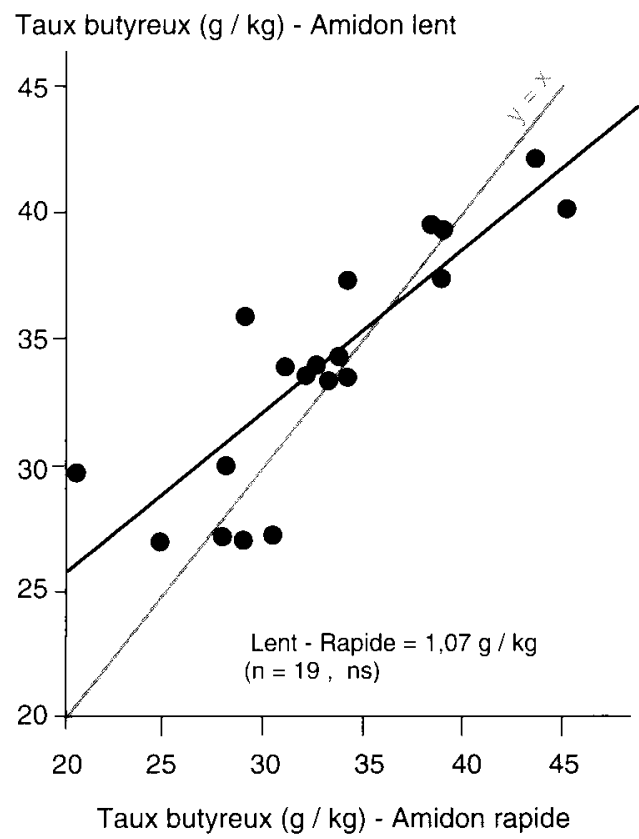


sa fraction amylacée présente une influence plus marquée. On est bien entendu tenté de rapprocher ce résultat de ce qui a été évoqué dans le paragraphe 2.2.c. à propos du profil fermentaire ruminal (figures 5 et 6). En effet on retrouve, avec le TB, le fait qu'un régime induisant des fermentations ruminales et un TB normal est peu sensible à la vitesse de dégradation des glucides. Les données rassemblées ne révèlent pas d'influence notable de la vitesse de dégradation de la fraction azotée. De même aucun résultat significatif n'apparaît comme conséquence du degré d'harmonisation entre les vitesses de dégradation des fractions glucidiques et azotées des aliments.

\section{3 / L'amortissement des phénomènes dynamiques digestifs}

Le manque de résultats convaincants sur l'influence du degré d'harmonisation des cinétiques de dégradation des glucides et de l'azote sur la protéosynthèse microbienne et les résultats zootechniques amène à s'interroger sur les mécanismes qui permettent d'amortir aussi efficacement les perturbations liées aux différences de vitesse de digestion de l'azote et des glucides. La finalité de ces mécanismes est vraisemblablement de pouvoir fournir en "bout de chaîne ", de façon quasi continue, des nutriments disponibles pour les synthèses cellulaires.

Les travaux cherchant à élucider spécifiquement les phénomènes d'amortissement dynamique, c'est-à-dire des phénomènes de retard ou de délai, sont encore rares. Ils concernent d'abord l'identification de structures permettant d'obtenir ces phénomènes de délai. Il s'agit en particulier d'identifier des structures compartimentales au sein des voies d'utilisation des nutriments. Ainsi la

Figure 11. Simulation du rôle d'un compartiment de délai sur la dynamique de la croissance microbienne.

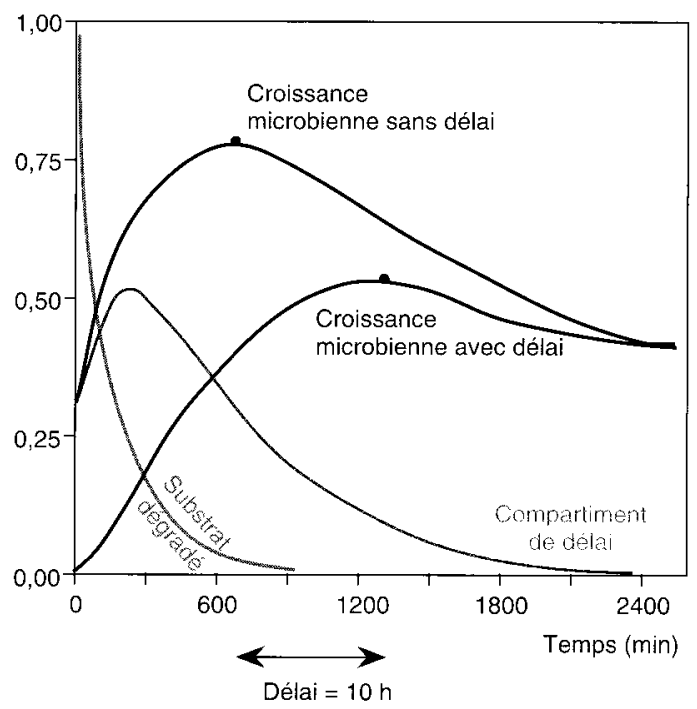

figure 11 illustre l'influence de l'existence, sur la dynamique de la croissance microbienne, d'un compartiment intermédiaire dans le cadre d'une modélisation simple de la digestion microbienne. Il apparait que, sous l'angle dynamique, la dégradation n'est pas équivalente à l'utilisation par les microbes. La phase de latence qui intervient avant le démarrage du processus de digestion des parois végétales (voir plus haut) peut aussi s'interpréter par l'existence d'un compartiment initial de "maturation " de la paroi avant que celle-ci ne devienne digestible (Van Milgen et al 1991). Dans la réalité les structures induisant des phénomènes de délai sont souvent plus compliquées; les principales d'entre elles qui existent au niveau du réticulo rumen seront évoquées. Ce sont essentiellement des recherches sur les variations post-prandiales des paramètres digestifs ruminaux qui ont permis de révéler des structures et mécanismes d'amortissement.

\section{1 / Le rôle des cinétiques d'ingestion}

Le principe de synchronisation des processus de dégradation des glucides et de l'azote évoqué plus haut (cf figure 3) suppose d'abord que l'aliment, ou la ration, considéré est "plongé » instantanément dans le réticulorumen. En réalité l'entrée des substrats est largement répartie, ou retardée, dans le temps par la cinétique d'ingestion. La forme de cette cinétique est assez caractéristique, elle a déjà été modélisée empiriquement par une fonction exponentielle $(\mathrm{Y}=\mathrm{a}[1-\mathrm{b} \cdot \exp (-\mathrm{ct})]$ chez le bovin (Faverdin 1985), chez l'ovin (Baumont 1989) et chez les caprins en milieu méditerranéen (Meuret 1989). D'après les valeurs expérimentales des paramètres $a, b, c$, le grand repas de fourrage constitue un premier phénomène de délai d'une durée de 1 à 3 heures.

\section{2 / Le rôle des oligomères intermédiaires}

La technique de dégradation in situ est loin d'intégrer tous les aspects dynamiques de la digestion ruminale des protéines, en particulier il est connu que le phénomène de protéolyse excède celui de l'hydrolyse et de la captation des peptides. Ceci se traduit par un accroissement en période post-prandiale de la concentration des peptides dans le réticulorumen, la teneur en $\mathrm{N}$ peptidique étant comparable à celle d'N ammoniacal. D'après les travaux de Russel et al (1983) et Chen et al (1987), l'existence de ces compartiments peptidiques correspondrait à un phénomène de délai moyen d'environ 6 heures avant l'utilisation effective d'azote par les micro-organismes. Les acides aminés libres contribuent vraisemblablement aussi à ce rôle d'amortissement dynamique puisque leur concentration s'accroît en phase post-prandiale de façon importante (figure 12). Le délai apparent associé à ce compartiment des acides aminés se situe entre 2 et 3 heures d'après les résultats de Craig et al (1987) et de Dhiman et Sat- 
ter (1994). Compte tenu de la concentration non négligeable des composants peptidiques et des acides aminés dans le jus ruminal, il apparaît qu'un délai de 8 à 9 heures peut vraisemblablement exister entre la dégradation ruminale d'une protéine in situ et la synthèse microbienne qui en découle.

Des phénomènes de délai existent également pour la digestion glucidique. En effet, si les glucides solubles sortent très rapidement des sachets de nylon, ils ne sont pas pour autant utilisés par les microbes. Ainsi, différents auteurs, en particulier Leedle et al (1986) sur bovins et Clapperton et Czerkawski (1969) sur ovins ont clairement observé un phénomène d'accumulation postprandiale des sucres solubles dans le rumen avec des rations qui étaient respectivement riches en maïs et en pulpe de betteraves. Jouany (1978) avait également montré l'existence de larges différences entre régimes dans l'aptitude à entraîner une accumulation ruminale de sucres solubles. D'après ces différentes données et d'autres essais publiés, les compartiments intermédiaires des sucres solubles pourraient traduire un phénomène de retard de l'ordre de 1,5 heure du processus d'utilisation de l'énergie par les microbes.

Figure 12. Variations post-prandiales des teneurs en acides aminés libres et en $\mathrm{NH}_{3}$ du jus de rumen en fonction du régime (Dhiman et Satter 1994).

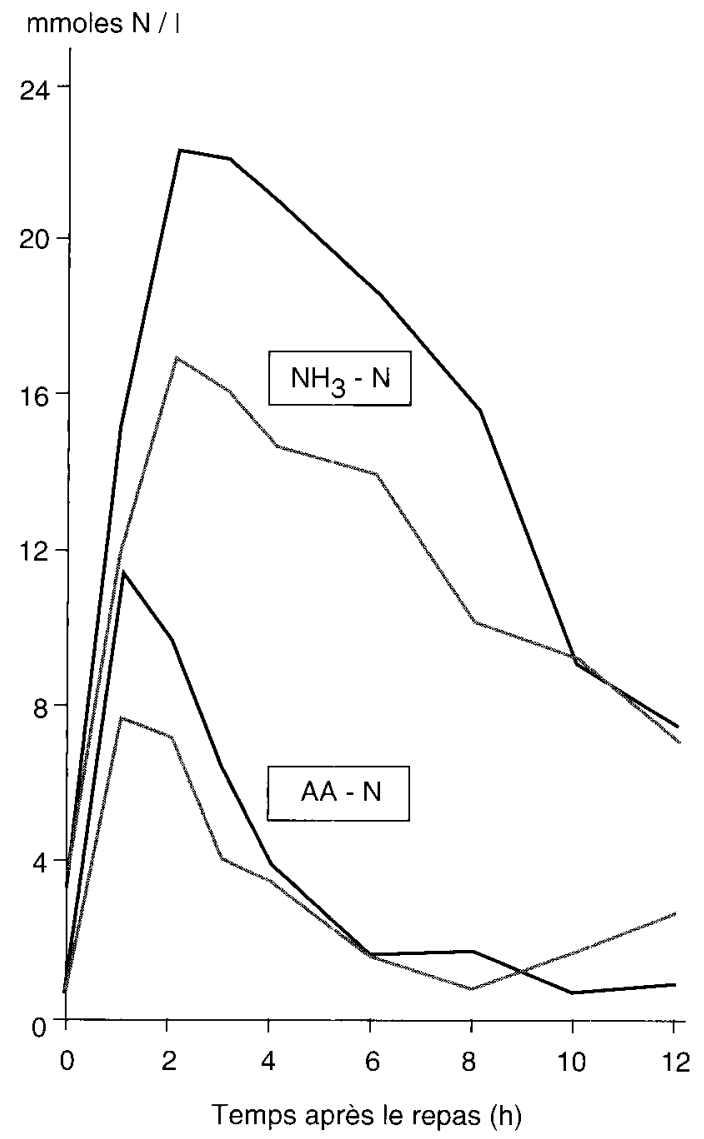

Ensilage de luzerne

$1 / 3$ ensilage de luzeme $+2 / 3$ ensilage de mais

\section{3 / La dynamique du métabolisme microbien}

Des études conduites in vitro par Newbold et Rust (1992) ont montré que, sur une durée de fermentation de 12 heures, les micro-organismes du rumen sont capables de compenser efficacement une asynchronie de la mise à disposition des nutriments azotés et glucidiques malgré le fait que ces irrégularités peuvent induire des phases transitoires de dépression de la croissance microbienne.

\section{a / La colonisation microbienne}

La colonisation microbienne des particules constitue un phénomène conditionnant la dégradation des substrats non solubles nouvellement arrivés. Cette colonisation débute très rapidement. Ainsi Yang (1991) a observé que $1 \%$ de la MS du substrat était colonisée par les microbes dans le premier quart d'heure de présence dans le rumen. Le taux de croissance de la colonisation des substrats est rapide, entre 10 et $30 \%$ par heure, c'està-dire bien plus élevé que le taux de croissance microbienne. Cependant, d'après les résultats expérimentaux de Yang (1991) et Van Milgen et al (1993), ce taux ne semble pas être lié au degré de synchronisation de la dégradation de l'azote et des glucides ni à la vitesse de dégradation de l'un de ces 2 constituants. Par contre, 3 heures après le début de l'incubation, la colonisation bactérienne est essentiellement liée à la teneur en paroi végétale (figure 13). Ceci traduit le rôle de surface d'adhésion important joué par les constituants pariétaux. Le niveau de colonisation maximum se situe vers $10-15 \%$ de la matière sèche résiduelle in situ. Des études conduites par Yang (1991) et par Van Milgen et al (1993), ont montré que la colonisation d'un "sous-système ruminal ", constitué par le contenu d'un sachet de nylon, passait par un maximum après 30 à 60 heures d'incubation. Ces cinétiques de colonisation de la matière sèche ou du contenu d'un sachet varient d'un aliment à l'autre mais, là encore, elles ne semblent pas être associées à l'ampleur des phénomènes de dégradation des fractions azotées et glucidiques. Il ressort donc de ces différents résultats que la dynamique de la colonisation microbienne ne serait pas liée à des variations de l'équilibre des fractions glucidiques et azotées disponibles.

\section{b / L'adaptation enzymatique}

Des études conduites au cours de la dernière décennie ont montré que les micro-organismes du rumen présentaient des cinétiques marquées d'activité enzymatique en fonction du temps après le repas et de la nature du régime (Williams et al 1989, Martin et al 1993). Le délai d'adaptation de cette activité enzymatique contribue vraisemblablement au délai global d'utilisation des substrats. 
Figure 13. Relation entre la teneur en paroi végétale et la colonisation microbienne d'un aliment après 3 heures d'incubation in situ (Yang 1991).

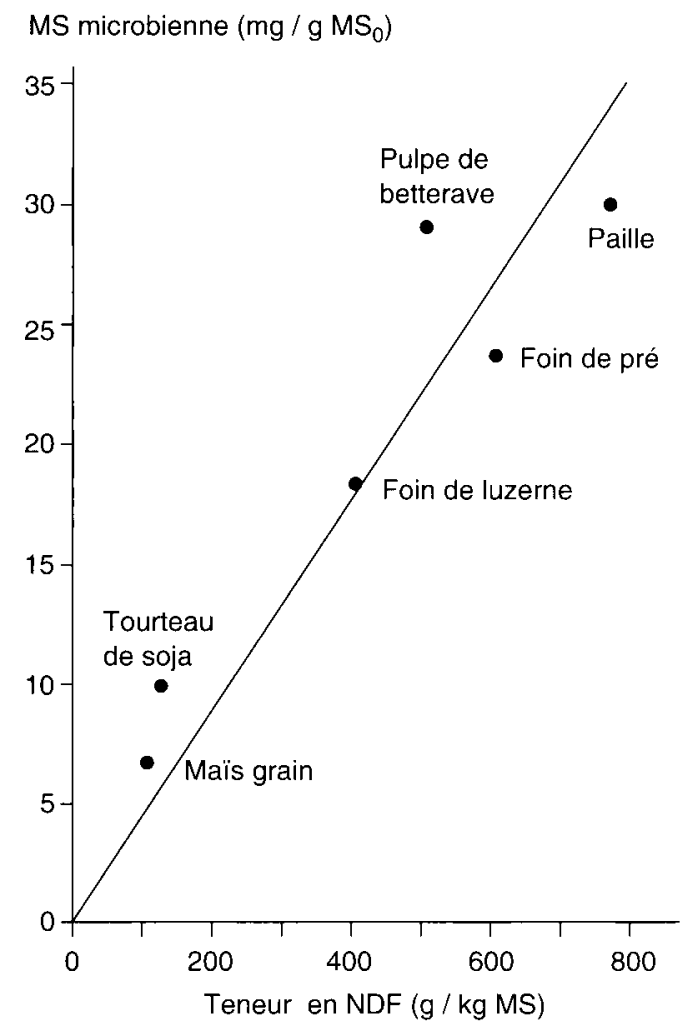

\section{c / Le rôle du stockage glucidique microbien}

Les micro-organismes du rumen contiennent 10 à $40 \%$ de leur matière sèche sous forme de polysaccharides. Cette teneur est positivement liée à la densité énergétique du régime ingéré (Mc Allan et Smith 1974) et à son rapport glucide/azote. Plusieurs auteurs ont également montré que cette réserve glucidique présentait des caractéristiques dynamiques marquées (figure 14). L'accroissement relatif de cette réserve est très rapide aussitôt après le repas ( 3 à $5 \% / \mathrm{min}$ ). Cette rapidité permet d'endiguer transitoirement une part significative du flux de carbone destiné à fabriquer des acides gras volatils, elle joue donc vraisemblablement un rôle de prévention de l'acidification post-prandiale du rumen. La rapidité et l'efficacité de ce processus est notamment permise par le fait que les protozoaires sont capables "d'avaler » directement de l'amidon et de créer ainsi un retard dans le déroulement des processus de dégradation et néosynthèse des chaînes carbonées. Une telle modalité d'utilisation ne semble pas être possible pour des substances telles que les pectines. Ceci explique vraisemblablement pourquoi des ingrédients tels que les pulpes d'agrumes ou de betteraves sont plus acidogènes que des céréales à amidon rapide (Michalet-Doreau et Sauvant 1989). Après le maximum atteint 1 à 2 heures après le début du repas, la réserve polysacchari- dique microbienne décroît plus lentement (5 à $20 \% / \mathrm{h}$ ) au rythme de l'utilisation de ce substrat par les cellules microbiennes. D'après les cinétiques les plus complètes de la littérature, le délai de l'utilisation des glucides, révélé par ce compartiment glucidique intermédiaire, peut-être important. On l'a ainsi estimé à $7 \mathrm{~h}$ pour des protozoaires avec un régime riche en orge (Jouany et Thivend $1972 \mathrm{a}$ et b) et à 8,9 et $14,1 \mathrm{~h}$ pour des bactéries respectivement libres et liées (Craig et al $1987 \mathrm{~b}$ ). D'après les simulations que nous avons effectuées, cette réserve glucidique pourrait être la source majeure d'énergie pour le métabolisme microbien entre 2 et $6 \mathrm{~h}$ après le début d'un repas.

\section{d / Le rôle des lipides des micro-organismes}

Il a été montré, en particulier à l'INRA (Legay-Carmier et Bauchart 1989), que les micro-organismes accumulaient de façon transitoire les lipides apportés par la ration. Il conviendrait de préciser le rôle de ce compartiment, en particulier de pouvoir quantifier l'ampleur et les propriétés dynamiques de ce stockage transitoire d'énergie disponible pour le métabolisme microbien.

\section{e / Dynamique fermentaire et stoechiométrie}

Les principales voies fermentaires de la digestion dans le rumen sont bien établies, en outre de nombreux essais ont permis de montrer que le profil fermentaire des AGV était pour partie dépendant du régime alimentaire. En particulier il est connu qu'un régime riche en aliments concentrés, ou riche en fourrage de bonne digestibilité, induit des fermentations ruminales plus intenses et, en général, plus "propioniques". Il est surprenant que ce constat, déjà ancien, n'ait toujours pas reçu d'hypothèse explicative mécaniste. Il faut pour cela, pensons-nous, chercher à mieux comprendre et intégrer les régulations de la répartition cellulaire des flux de carbone issus de la dégradation des substrats entre, d'une part, l'élaboration de biomasse microbienne et la synthèse des produits terminaux des fermentations et, d'autre part, leur répartition entre ces produits terminaux : l'équilibre gaz-AGV ainsi que l'équilibre des $\mathrm{AGV}$ entre eux. Cette répartition des flux s'appelle stœchiométrie.

Il semble que les régulations impliquées soient largement dépendantes du statut énergétique des cellules microbiennes et de la disponibilité en hydrogène du milieu ruminal. En effet les cellules microbiennes paraissent disposer, à certains moments, d'un excès de chaînes carbonées, par rapport à leur capacité de stockage (cf. ci-dessus), et de synthèse de biomasse nouvelle. Il y a alors un excès potentiel d'énergie disponible qui peut d'ailleurs être évalué par la mesure de la teneur en ATP du milieu (Erfle et al 1981). Dans une telle situation, les cellules micro- 
biennes mettent en jeu des régulations qui tendent à favoriser les voies métaboliques qui sont moins efficaces dans l'élaboration d'ATP. Ainsi les AGV à chaîne plus longue fournissent moins d'ATP par carbone terminal. En conséquence, la présence, en proportion plus importante d'AGV à chaînes carbonées à 3,4 , 5 ou 6 atomes de carbone, serait un indicateur de l'existence d'un excès relatif d'énergie disponible par rapport à la capacité de synthèse microbienne. Ce phénomène peut s'observer à partir des résultats publiés dans la littérature; ainsi un régime plus riche en concentré, donc en énergie disponible, favorise systématiquement les AGV à chaînes carbonées à plus de 2 atomes de carbone, les acides propionique et butyrique, ce qui est connu, mais également les acides gras mineurs à 5 ou 6 atomes de $\mathrm{C}$ (figure 15). Les figures 5 et 6 , évoquées dans le paragraphe 2.2.c, indiquent que les différences de vitesse de dégradation des glucides de la ration influencent le profil des AGV, de façon cohérente avec ces principes. Les amidons rapides semblent donc aboutir à des situations d'excès transitoires d'énergie pour les micro-organismes du rumen, particulièrement si l'ensemble de la ration est déjà facilement digestible (figures 5 et 6 ).
Figure 15. Influence de la proportion d'aliments concentrés dans le régime sur la proportion d'acides gras mineurs (acides en C5 et C6) dans le jus de rumen (Lescoat et Sauvant 1995).

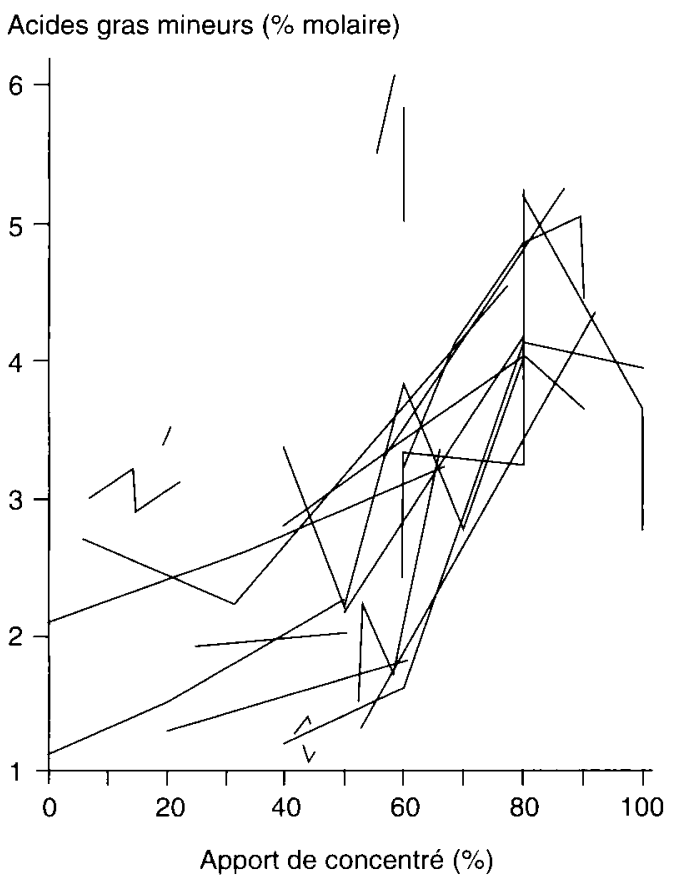

Figure 14. Evolution de la teneur en polysaccharides des microbes du rumen après un repas.

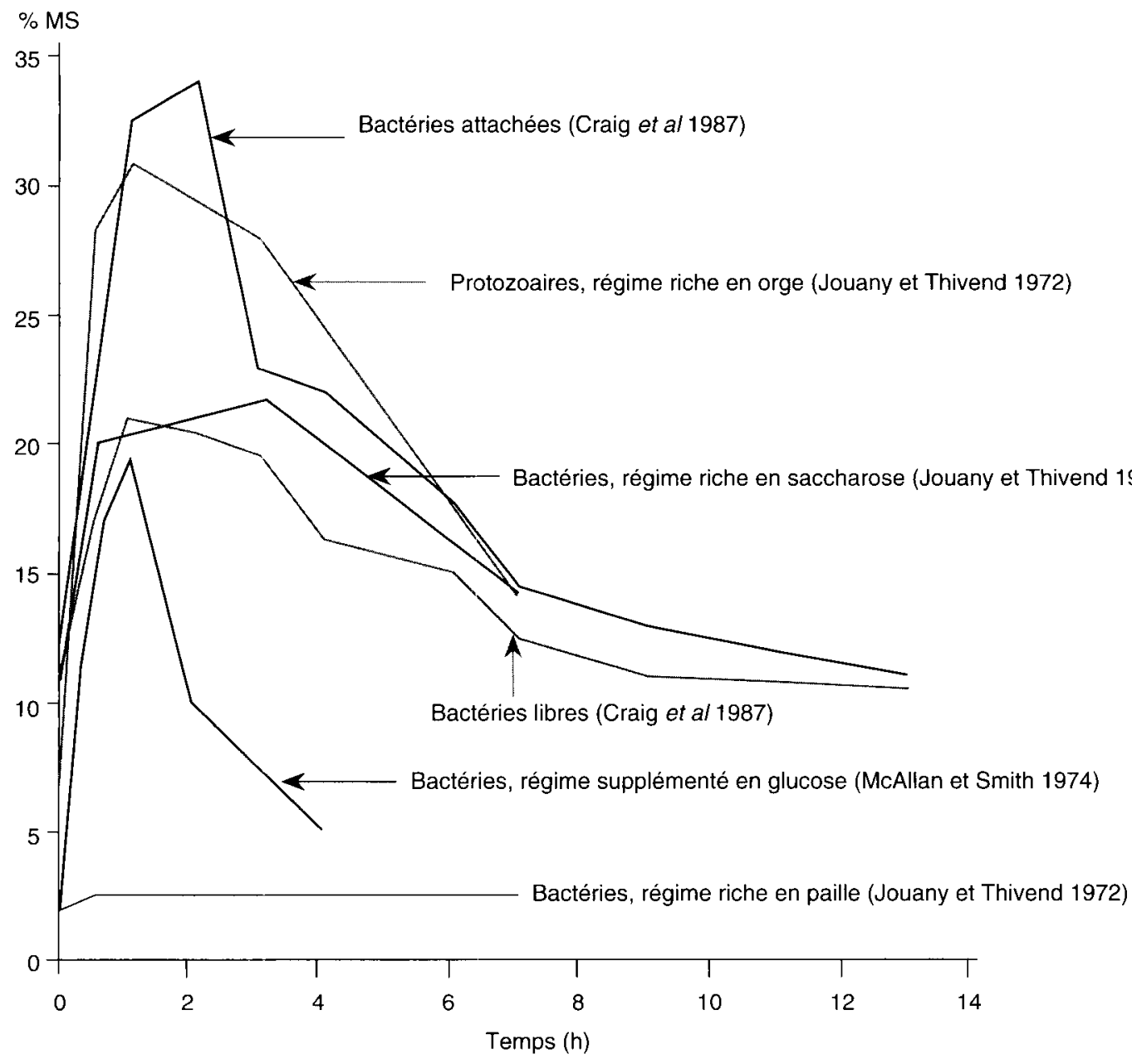


Les phénomènes de recyclage permettent également de mieux répartir dans le temps la fourniture de nutriments azotés aux microbes du rumen.

\section{f / Limite de la capacité de croissance et de transit des microbes}

Il convient de chercher à mieux comprendre pourquoi la capacité de croissance microbienne semble "plafonner " avec des rations où les chaînes carbonées et l'azote disponible ne sont pas facteurs limitants. Cette situation se rencontre par exemple avec les régimes riches en glucides et en azote rapidement dégradable. Ce phénomène n'est pas négligeable puisqu'il apparaît clairement lorsque l'on regroupe des essais publiés sur les variations de la synthèse microbienne en fonction de la proportion d'aliments concentrés dans le régime (figure 16). Cet effet ne semble pas être la conséquence d'une limitation de la capacité fermentaire des microbes. Il apparaît plutôt comme une incapacité métabolique à synthétiser de nouvelles cellules, le temps moyen de division ne semblant pas pouvoir être inférieur à 5-6 heures. On peut penser que cette limitation de la capacité synthétique des microbes pourrait être la conséquence de rétroactions négatives induites par l'accumulation de produits intermédiaires (acides aminés...) ou terminaux (AGV, hydrogène...) et/ou par certains paramètres physico-chimiques de la niche écologique ruminale. Linfluence négative d'un $\mathrm{pH}$ acide correspond à l'effet le plus connu mais il est vraisemblable que d'autres paramètres tels que la pression osmotique, le potentiel redox... peuvent également jouer un rôle inhibiteur de l'activité microbienne. Un autre facteur important peut également contribuer à expliquer ce phénomène. La synthèse microbienne ruminale est évaluée indirectement à travers des mesures de flux duodénal. Or avec des rations riches en concentrés, le flux

Figure 16. Influence de la proportion d'aliments concentrés dans le régime sur la synthèse microbienne ruminale (Munoz et al 1995).

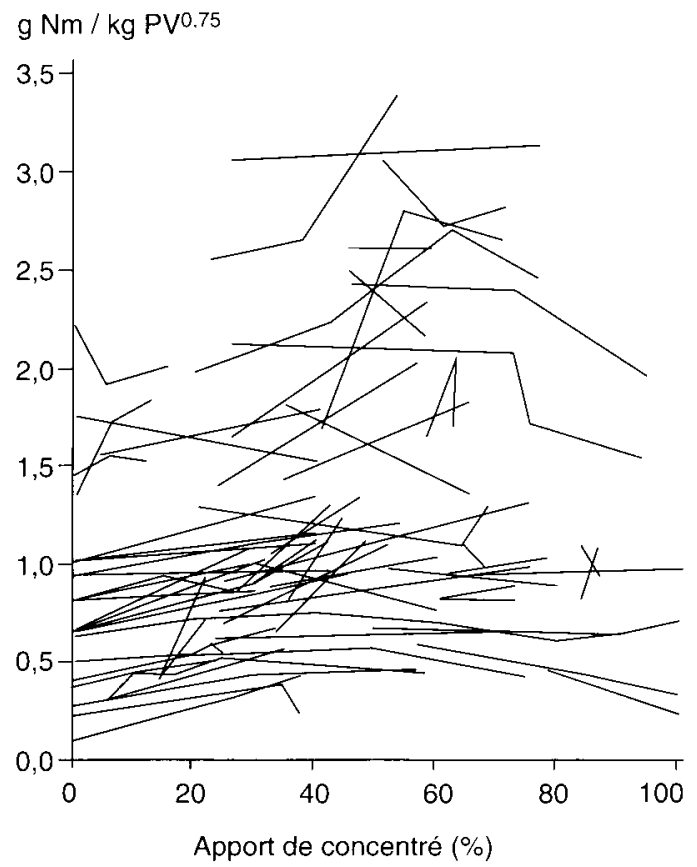

duodénal de matière sèche d'origine alimentaire diminue sensiblement. Donc, compte tenu du fait que la majeure partie de la population microbienne est attachée aux particules, l'accroissement de la proportion de concentré dans le régime tend à limiter le flux du support permettant la sortie des micro-organismes du rumen. Compte tenu de l'importance des conséquences pratiques des variations du flux protéique microbien intestinal, il conviendrait de pouvoir renforcer les recherches dans ce secteur.

\section{4 / Le rôle dynamique des protozoaires}

Plusieurs revues de la littérature ont permis d'avoir une idée globale du rôle des protozoaires dans la digestion microbienne à partir des effets moyens associés à la défaunation (Williams et Coleman 1988, Ushida et al 1991). Il est en particulier établi qu'ils ont, par leur présence, une contribution positive à la dégradation des substrats et qu'ils entraînent une ammoniogénèse accrue dans le rumen. Il n'y a par contre pas ou peu d'étude spécifique sur le rôle dynamique des protozoaires. Outre le rôle d'amortissement dynamique énergétique que peut jouer leur stockage glucidique évoqué plus haut, il est vraisemblable que les protozoaires constituent un compartiment de délai par dérivation particulièrement important pour l'azote. En effet, les protozoaires se caractérisent par une activité de prédation importante de bactéries (de 40 à $80 \%$ des bactéries disparaissant) sachant qu'ils s'écoulent apparemment très peu par transit hors du rumen (demi-vie de l'ordre de $100 \mathrm{~h}$ ) et que, de ce fait, leur biomasse doit être largement recyclée sous forme de protéines ou de peptides disponibles dans le milieu ruminal.

\section{5 / Le rôle des phénomènes de recyclage}

La physiologie digestive du ruminant se caractérise par une forte capacité à recycler certains nutriments dans la partie antérieure de leur tube digestif. Cela concerne en particulier les tampons minéraux (bicarbonate...) et l'urée. On commence à mieux comprendre les aspects dynamiques quantitatifs du recyclage de l'urée, grâce en particulier aux travaux de Rémond et al (1993) sur des animaux cathétérisés de la veine ruminale. Il apparaît que le repas se traduit par une phase précoce d'accumulation ruminale et d'absorption d'ammoniaque. Cette vague est endiguée par le foie qui transforme, avec un délai qu'on peut estimer à 2-3 h, l'ammoniaque en urée. L'augmentation consécutive de l'urémie, moins forte que celle d'NH $\mathrm{NH}_{3}$ ruminal en raison de la différence de taille entre les compartiments aqueux ruminaux et corporels, se traduit par une "vague" de son recyclage dans le tube digestif (figure 17). Ce processus met bien en évidence le rôle de "vases communicants" entre les compartiments aqueux cités vis-à-vis du métabolisme de l'ammoniaque et 
de l'urée. Sachant que, dans ce cas, le phénomène d'amortissement est principalement permis par le fait que le volume aqueux corporel est bien plus important $(\times 3)$ que le ruminal. Sur la base des résultats expérimentaux évoqués et des simulations que nous avons pu effectuer, ce recyclage d'urée permet de refournir de l'azote disponible aux micro-organismes dans une période située entre 3 et $8 \mathrm{~h}$ après un repas, période où le flux d'azote d’origine alimentaire est généralement réduit.

\section{Conclusions}

Il est maintenant bien établi que les cinétiques de dégradation des aliments ou de leurs constituants dans le rumen peuvent varier très largement en fonction de la nature de l'aliment et du traitement technologique qu'il a reçu. Sur la base de cette connaissance, il est possible de formuler des aliments, ou des régimes, caractérisés par des vitesses de dégradation plus ou moins rapides ou par une meilleure association entre les dynamiques de dégradation des glucides et de l'azote.

Les données publiées ces dernières années, voire ces derniers mois, permettent d'établir l'existence d'une influence de la cinétique de dégradation des fractions glucidiques amylacées sur la nutrition et les performances des ruminants, en particulier des vaches laitières. Les amidons lents confèrent une meilleure ingestibilité aux rations, ils conduisent à des fermentations ruminales plus régulières lorsque le régime alimentaire considéré riche en concentrés représente un excès énergétique pour les micro-organismes de la panse et, de ce fait, un faciès fermentaire propionique. Cet effet semble se répercuter sur le taux butyreux du lait. En effet celui-ci est également sensible à la vitesse de dégradation de l'amidon dans le cas de rations induisant un faible taux butyreux. Il convient cependant de souligner que les régimes induisant des fermentations ruminales et des valeurs de TB normales ne sont pas sensibles aux variations de la vitesse de dégradation de la fraction amylacée. Sur l'aspect azoté, l'emploi d'amidon à dégradation lente semble réduire de façon non négligeable le niveau d'apport de PDIM de la ration. Les données expérimentales publiées ne révèlent pas d'impact de cet effet sur le taux protéique du lait ; cependant, les rations utilisées étaient globalement riches en azote. Il convient donc de se demander si l'emploi d'amidons lents avec des rations plus limitées en azote (pour réduire les rejets d'urée urinaire) ne pourrait pas avoir d'effet défavorable sur la nutrition azotée voire le TP du lait. Des recherches sont nécessaires sur ce sujet.

Les données publiées ne permettent pas de démontrer l'intérêt technique de chercher à harmoniser les cinétiques de dégradation des fractions glucidique et azotée des aliments. Cette absence d'effet résulte peut-être en partie de la difficulté à le mettre en évidence expérimentalement. Elle résulte vraisembla-
Figure 17. Flux d'ammoniaque et d'urée à travers la paroi du rumen chez le mouton (régime : foin de dactyle en 2 repas par jour; Rémond et al 1993).

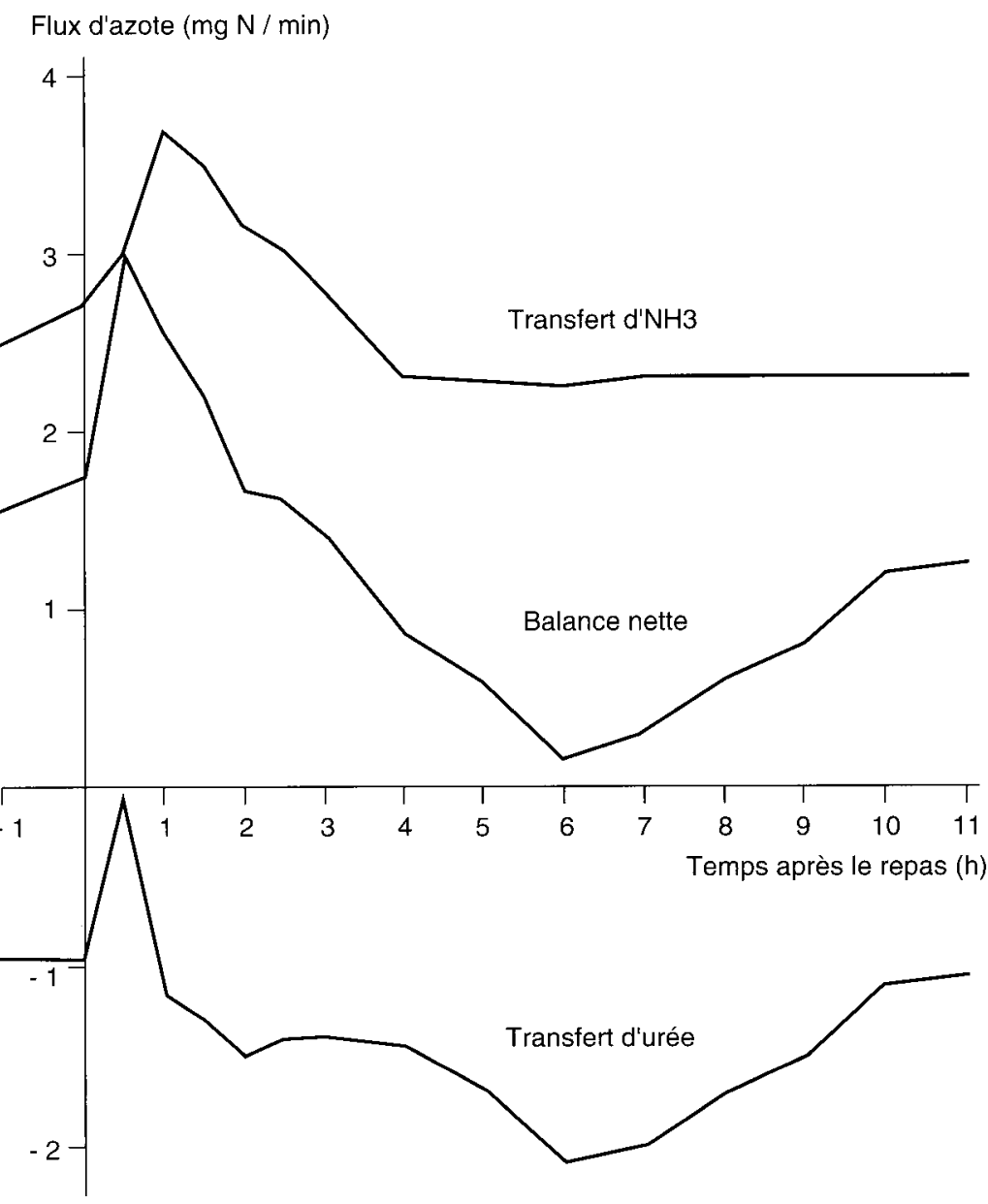

blement aussi de l'extraordinaire capacité du complexe digestif ruminal à «tamponner » les variations dynamiques des phénomènes ingestifs et digestifs. Il apparaît que cette capacité est la conséquence de la présence de structures compartimentales favorables à la mise en place de multiples phénomènes de délai et de recyclage dont les plus connus ont été évoqués. L'étude de ces phénomènes permet non seulement d'identifier les compartiments impliqués, mais également les flux dont le débit est limité. Parmi les flux qui présentent par leur limitation un rôle dans l'amortissement dynamique, il convient de souligner la capacité des microbes à capter les nutriments disponibles dans le liquide ruminal, et également leur capacité à synthétiser de la biomasse nouvelle. Ces limites se traduisent par des accumulations transitoires, d'une part, de nutriments glucidiques et surtout protéiques, dans le jus ruminal et, d'autre part, de polysaccharides dans les cellules microbiennes. Il y a quelques années on parlait de "vache accordéon " pour symboliser le rôle de tampon d'énergie joué par les réserves adipeuses à long terme pendant le cycle de production, peut-être pourrait-on maintenant parler de "rumen ou de microbes accordéons " pour exprimer le rôle de tampon dynamique joué dans le court terme par le rumen! 
D'un point de vue pratique, ces différents résultats confirment l'intérêt d'aborder les phénomènes digestifs et nutritionnels et l'alimentation sous l'angle dynamique. En effet même si la capacité de l'animal et de son rumen à amortir les phénomènes dynamiques amène à nuancer l'opportunité de certaines pratiques actuelles de formulation des régimes alimentaires, il faut considérer que ces connaissances ouvrent simultanément de nouvelles perspectives de recherche et d'innovation pour l'alimentation des ruminants.

Texte inspiré du rapport intitulé " Dynamic aspects of carbohydrate and protein breakdown and the associated microbial metabolism " présenté par les mêmes auteurs au VIII International Symposium of Ruminant Physiology (WILLINGEN, Allemagne).

\section{Références bibliographiques}

Aldrich J.M., Muller L.D., Varga G.A., Griel L.C., 1993. Nonstructural carbohydrate and protein effects on rumen fermentation, nutrient flow and performance of dairy cows. J. Dairy Sci., 77, 10911105.

Archimède H., Sauvant D., Dorléans M., Chapoutot P., Poncet C., 1995. Influence of the nature of forage and concentrate on the digestive interactions measured in sacco and in vivo. Anim. Feed Sci. Technol (in press).

Baumont R., 1989. Etat de réplétion du réticulorumen et ingestion de fourrages. Thèse de Doctorat INA-PG-Paris.

Chapoutot P., Giger S., Sauvant D., Jeantet S., 1990. Etude de l'additivité de la dégradation in sacco de la matière sèche des mélanges simples d'aliments concentrés. Reprod. Nutr. Develop., 30, $169-170$

Chapoutot P., Sauvant D., Dorléans M., 1993. Etude des paramètres de la dégradation dans le rumen des glucides pariétaux des aliments concentrés. Ann. Zootech., 42, 136-137.

Chen G., Russel J.B., Sniffen C.J., 1987. A procedure for measuring peptides in rumen fluid and data suggesting that peptide uptakes is a rate limiting step in ruminal protein degradation. J. Dairy Sci., 70, 1211-1219

Clapperton J.L., Czerkawski J.W., 1969. Methane production and soluble carbohydrates in the rumen of sheep in relation to the time of feeding and the effects of short term intra ruminal infusions of unsaturated fatty acids. Br. J. Nutr., 23, 813-816.

Craig W.N., Brown D.R., Broderik G.A., Ricker D.B., 1987a. Post prandial compositional changes of fluid and particle associated ruminal microorganisms. J. Anim. Sci., 65, 1042-1048.

Craig W.N., Broderick G.A., Ricker D.B., 1987b. Quantitations of microorganisms associated with the particulate phase of ruminant ingesta. J. Nutr., $117,56-62$

Dhiman T.R., Satter L.D., 1994. Milk yield and rumen fermentation measurements in cows fed diets containing different proportions of alfalfa and corn silage. J. Dairy Sci., 77, 222

Erfle J.D., Mahadevan S., Sauer F.D., 1981. Relationship between adenylate energy charge, rumen volatile fatty acid concentrations and rates of production and dry matter digestibility in the cow. J. Dairy Sci., 64, 634-642.

Faverdin P., 1985. Régulation de l'ingestion des vaches laitières en début de lactation. Thèse de Doctorat. INAPG-Paris.
Giger-Reverdin S., Munoz L.S., Sauvant D., Leboute E., Chapoutot P., 1993. Influences du degré d'harmonie des cinétiques de dégradation des fractions glucidiques et azotées des aliments concentrés sur les fermentations en Rusitec. Ann. Zootech., 42, 148149 .

Herrera-Saldana R., Huber J.T., 1989. Influence of varying protein and starch degradabilities on performance of lactating cows. J. Dairy Sci., 72, 1477 1483 .

Jarrige R., 1981. Les constituants glucidiques des fourrages : variations, digestibilité et dosage. In : C. Demarquilly (ed), Prévision de la valeur des aliments des ruminants, 13-40. INRA Editions, Paris.

Jouany J.P., Thivend P., 1972a. Evolution post-prandiale de la composition glucidique des corps microbiens du rumen en fonction de la nature des glucides du régime. I. les protozoaires. Ann. Biol. anim. Bioch. Biophys., 12, 673-677.

Jouany J.P., Thivend P., 1972b. Evolution post pandiale de la composition glucidique des corps microbiens du rumen en fonction de la nature des glucides du régime. II. les bactéries. Ann. Biol anim. Bioch. Biophys., 12, 679-683.

Kung L., Tung R.S., Carmean B.R., 1992. Rumen fermentation and nutrient digestion in cattle fed diets varying in forage and energy source. Anim. Feed Sci., 39, 1-12.

Leedle J.A.Z., Barsuhn K., Hespell R.B., 1986. Post prandial trends in estimated ruminal digesta polysaccharides and their relations to changes in bacterial groups and ruminal fluid characteristics. J. Anim. Sci., 62, 789-803.

Legay-Carmier F., Bauchart D., 1989. Distribution of bacteria in the rumen contents of dairy cows given a diet supplemented with soya bean oil. Br. J. Nutr., 61, 725-740.

Lescoat P., Sauvant D., 1995. Developpement of a mechanistic model for rumen digestion validated using the duodenal flux of animo acids. Reprod. Nutr. Develop. 35, 45-70.

Madsen J., 1985. The basis of the proposed nondic potein evaluation system for ruminants. The AAT PBV system. Acta Agric. Scand. Suppl., 25, 9-20.

Martin C., Michalet-Doreau B., Fonty G., Williams A., 1993. Post prandial variations in the activity of polysaccharide degrading enzymes of fluid and particle associated ruminal microbial population. Curr. Microb., 27, 223-228.

Martin C., 1994. Influence du pH ruminal sur la digestion des parois végétales, en relation avec les modifications de l'activité fibrolytique de l'éco-sys- 
tème microbien. Thèse de l'université de ClermontFerrand.

Mc Allister T.A., Rode L.R., Cheng K.J., BuchananSmith J.G., 1989. The effect of formaldehyde on the in vitro digestion of barley starch. A.J.A.S., 2, 335336 .

Mc Allan A.R., Smith R.H., 1974. Carbohydrate metabolism in the rumen; bacterial carbohydrates formed in the rumen and their contribution to digesta entering the duodenum. Br. J. Nutr., 31, 77-88.

Mc Carthy R.D., Klusmeyer T.H., Vicini J.L., 1989. Effect of source of protein and carbohydrate on ruminal fermentation and passage of nutrients to the small intestine of lactating cows. J. Dairy Sci., 72, 2002-2016.

Meuret M., 1989. Valorisation par des caprins laitiers de rations ligneuses prélevées sur parcours. Thèse de la Faculté des Sciences Agronomiques de Gembloux.

Michalet-Doreau B., Sauvant D., 1989. Influence de la nature du concentré, céréale ou pulpe de betterave, sur la digestion chez les ruminants. INRA Prod. Anim., 2, 235-244.

Newbold J.R., Rust S.R., 1992. Effect of asynchronous nitrogen and energy supply on growth of ruminal bacteria in batch culture. J. Anim. Sci., 70, 538546.

Rémond D., Chaise J.P., Delval E., Poncet C., 1993. Net flux of metabolites across the ruminal wall of sheep fed twice a day with orchand grass hay. J. Anim. Sci., 71, 2529-2538.

Russel J.B., Sniffen C.F., Van Soest P.J., 1983. Effect of carbohydrate limitation on degradation and utilization of casein by mixed rumen bacteria. J. Dairy Sci., 66, 763-775.

Sauvant D., Giger S., 1987. Straw digestibility calculation and digestive interactions, Proc. EEC Seminar, COST 84, "Evaluation of straw as ruminant feed ". Ed. EEC.

Sauvant D., Chapoutot P., Archimède H., 1994. La digestion des amidons par les ruminants et ses conséquences. INRA Prod. Anim., 7, 115-124.
Sinclair L.A., Gainsworthy P.C., Newbold J.R., Buttery P.J., 1993. Effect of synchronizing the rate of dietary energy and nitrogen release on rumen fermentation and microbial protein synthesis in sheep. J. Agric. Sci., Camb., 120, 251-263.

Sniffen C.J., Van Sœest P.J., Fox D.J., O'connor J.D., Russell J.B., 1992. A net carbohydrate and protein system for evaluating cattle diets, II Carbohydrate and nitrogen availability. J. Anim. Sci., 70, 3582.

Tamminga S., Van straalen W.M., Subnel A.P.J., Meijer R.G.M., Steg A., Wever C.J.G., Block M.C., 1994. The dutch protein evaluation system : the DVE/OEB system. Livest. Prod. Sci., 40, 139-145.

Ushida K., Jouany J.P., Demeyer D.J., 1991. Effects of presence or absence of rumen protozoa on the efficiency of utilization of concentrate and fibrous feed. In : Tsuda T., Sasaki Y., Kawashira R. (eds), Physiological aspects of digestion and metabolism in ruminants, 625-654. Proc. of the 7th International Symposium on Ruminant Physiology.

Van Milgen J., Murphy M.R, Berger L.L., 1991. A compartimental model to analysis ruminal digestion. J. Dairy Sci., 74, 2515-2529.

Van Milgen J., Berger L.L., Murphy M.R., 1993. An integrated dynamic model of feed hydration and digestion and subsequent bacterial mass accumulation in the rumen. Br. J. Nutr., 70, 471-483.

Vérité R., Michalet-Doreau B., Chapoutot P., Peyraud J.L., Poncet C., 1987. Révision du système des protéines digestibles dans l'intestin. Bull. Tech. CRZV Theix, INRA, 70, 19-34.

Williams A.G., Coleman G.S., 1988. The rumen protozoa p. $77-128$ in the rumen microbial ecosystem, Ed. Hobson P.N., Elsevier.

Yang W., 1991. Etude cinétique de la colonisation microbienne des aliments dans le rumen du mouton, conséquences sur la compartimentation de la biomasse et sur sa dynamique de sortie du rumen dans le cas de différents types de rations. Thèse de Doctorat, Université de Clermont-Ferrand.

\section{Consequences of kinetics of ruminal degrada- tion on ruminal metabolism and zootechnical performances.}

Research of the last decades have demonstrated that the kinetics of ruminal degradation of feeds vary largely in function of their nature and of the constituent. It can therefore be envisaged to take the opportunity of this variability to formulate compound feeds or mixed diets which are more and less rapidly degraded or more and less synchronized between the carbohydrates and proteins degradation rates in comparison with the microbe requirements.

Experimental data related to the influence of the variations of these phenomena on ruminal digestion and zootechnical performances show that the effects are less important that could be a priori imagined. It is therefore necessary to explain that fact. It seems that numerous struc- tures and phenomena of delay allow to smooth efficiently the dynamic variations of degradation processes between feeds or constituents. Several examples are evoked. For nitrogenous products smoothing phenomena particularly occur by a transient storage of peptides before microbial captation and nitrogenous recycling by protozoa and blood urea recycling. For energy nutrients, it seems that the most important transient storage of energy is in the form of polysaccharidic storage within the microbial cells. The dynamic approach of ruminal digestion also emphasizes that microbial growth rate is limited and less variable in short term, even if sufficient nutrients are available. Some comments and explanations are made on this aspect.

SAUVANT D., VAN MILGEN J., 1995. Les conséquences de la dynamique de la digestion des aliments sur le métabolisme ruminal et les performances animales. INRA Prod. Anim., 8 (5), 353-367. 